\title{
Políticas públicas em infecções sexualmente transmissíveis no Brasil
}

doi: 10.1590/S1679-4974202100019.esp1

Public policies on sexually transmitted infections in Brazil

Políticas públicas sobre infecciones de transmisión sexual en Brasil

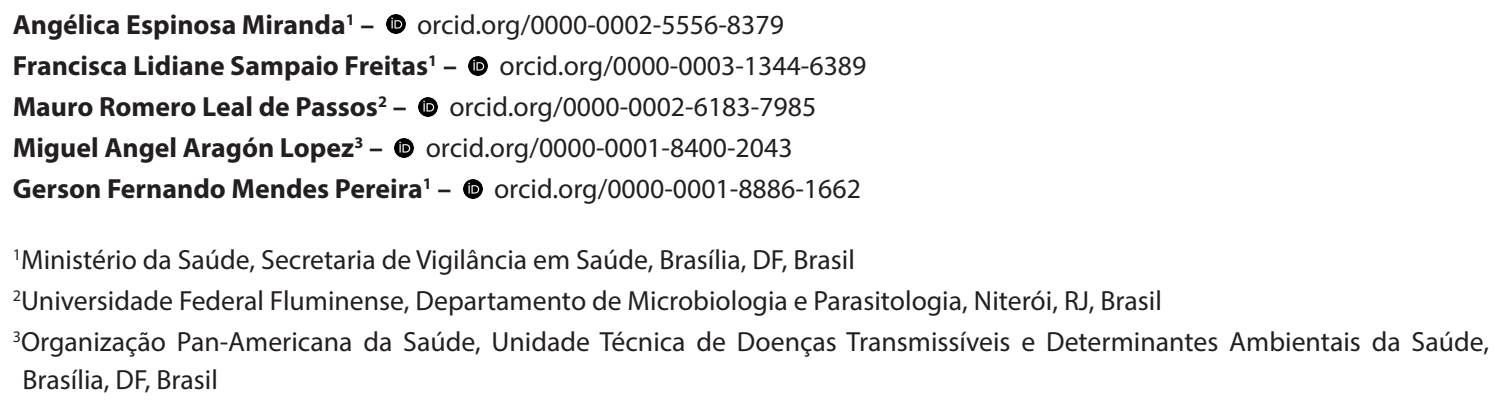

\section{Introdução}

Este artigo apresenta breve histórico de políticas públicas de enfrentamento às infecções sexualmente transmissíveis (IST) no Brasil. Ele também aborda lacunas e desafios a serem considerados pelo país, na formulação e implementação de políticas vindouras.

\section{Panorama global, da Região das Américas e do Brasil}

As IST persistem como problema de Saúde Pública mundial. Em 2016, a Organização Mundial da Saúde (OMS) estimou uma incidência de 376,4 milhões de casos de IST curáveis em pessoas de 15 a 49 anos de idade, entre os quais destacaram-se 127,2 milhões de casos de clamídia, 86,9 milhões de casos de gonorreia e 6,3 milhões de casos de sífilis. Na Região das Américas, estimaram-se 29,8 milhões de casos de clamídia, 13,8 milhões de casos de gonorreia e 2 milhões de casos de sífilis. ${ }^{1}$

Tais estimativas indicam alta frequência das IST e justificam a estratégia global da OMS e a definição de ações prioritárias para alcançar metas de eliminação das IST até 2030. Essa estratégia concentra esforços (i) na resistência antimicrobiana do gonococo e no risco de coinfecção por clamídia, (ii) na eliminação da sífilis congênita, o que requer ampliação de testagem e tratamento de gestantes e populações específicas, e (iii) na infecção pelo papilomavírus humano (buman papilomavirus, HPV), com ênfase em imunização para eliminação do câncer do colo de útero e verrugas anogenitais. $^{2}$

Endereço para correspondência:

Angélica Espinosa Miranda - Ministério da Saúde, Secretaria de Vigilância em Saúde, SRTVN, Quadra 701, Via W 5 Norte, Lote D, Edifício PO 700, Brasília, DF, Brasil. CEP: 70719-040

E-mail: angelica.miranda@aids.gov.br 
0 Plano de Ação para Prevenção e Controle do HIV/IST (2016-2021) da Organização Pan-Americana da Saúde (OPAS) propõe acelerar a eliminação das epidemias do vírus da imunodeficiência humana (buman immunodeficiency virus, HIV), e das IST como problemas de Saúde Pública na Região das Américas, até 0 ano de $2030 .{ }^{3}$ Esse planejamento inclui a estratégia regional para eliminação da transmissão vertical de HIV e da sífilis congênita, com critérios e indicadores específicos.

No Brasil, os casos de sífilis adquirida, sífilis em gestantes e sífilis congênita são de notificação compulsória. ${ }^{4}$ Dados epidemiológicos nacionais destacam o aumento de casos de sífilis entre 2010 e 2018, período em que a taxa de incidência de sífilis congênita aumentou quase quatro vezes, passando de 2,4 para 9,0 casos por mil nascidos vivos, e a taxa de detecção de sífilis em gestantes aumentou cerca de seis vezes, passando de 3,5 para 21,4 casos por mil nascidos vivos. A sífilis adquirida teve sua taxa de detecção aumentada de 34,1 casos por 100 mil habitantes, em 2015, para 75,8 casos por 100 mil habitantes em 2018.5

Estudos sobre prevalência de IST no Brasil com representatividade nacional revelam a magnitude do problema. Parturientes atendidas em maternidades públicas apresentaram uma prevalência de $9,8 \%$ de clamídia e 1,0\% de gonorreia em $2011,{ }^{6}$ e homens que procuraram atendimento em clínicas de IST em 2005, 13,1\% de clamídia e 18,4\% de gonorreia. ${ }^{7}$ Em 2015, mulheres vivendo com HIV apresentaram prevalência de $2,1 \%$ de clamídia e $0,9 \%$ de gonorreia, ${ }^{8}$ e $28,4 \%$ de HPV de alto risco. ${ }^{9}$ Em 2017, a prevalência de infecção por HPV foi de 25,4\% em colo de útero, 36,2\% na região peniana, $25,7 \%$ na região anal e $11,9 \%$ na região oral. ${ }^{10}$ Outro estudo nacional relatou a prevalência de $0,6 \%$ de sífilis em conscritos. ${ }^{11} \mathrm{Em} 2016$, maiores prevalências de sífilis foram observadas em segmentos de populações-chave, como homens que fazem sexo com homens $(9,9 \%),{ }^{12}$ trabalhadoras do sexo $(8,5 \%)^{13}$ e pessoas privadas de liberdade $(3,8 \%) .{ }^{14}$

\section{Histórico da resposta às IST no Brasil}

A Figura 1 resume os marcos históricos das principais estratégias e ações no âmbito das políticas públicas em IST no Brasil, a partir da criação do Programa Nacional de Doenças Sexualmente Transmissíveis e Aids (PN-DST/Aids) em 1986.
Destaca-se a publicação, no ano de 2015, do primeiro Protocolo Clínico e Diretrizes Terapêuticas (PCDT) para Atenção Integral às Pessoas com IST, aprovado pela Comissão Nacional de Incorporação de Tecnologias no Sistema Único de Saúde (Conitec). ${ }^{15}$ 0 documento define critérios para diagnóstico, preconiza tratamento, estabelece mecanismos de controle clínico - a serem seguidos pelos gestores do Sistema Único de Saúde (SUS) -, baseia-se em evidência científica e avalia parâmetros de eficácia, segurança, efetividade e custo-efetividade das tecnologias recomendadas. ${ }^{16}$ Naquela publicação, inaugurou-se a substituição do termo doença sexualmente transmissível por IST, permitindo-se alinhar à designação da OMS ${ }^{3}$ e assim, chamar a atenção do país para as infecções assintomáticas e não apenas a doença com sinais e sintomas. Em 2018, o PCDT foi novamente revisado e aprovado pela Conitec. ${ }^{17}$

Entre 2015 e 2016, a parceria do Ministério da Saúde com a Universidade Federal de Santa Catarina e sítios sentinelas, para o desenvolvimento de estudo sobre resistência do gonococo (Projeto SenGono), viabilizou o primeiro programa nacional de vigilância de susceptibilidade do gonococo aos antimicrobianos. A pesquisa de vigilância, de âmbito nacional, analisou cepas de gonococo em sete sítios sentinelas (compostos por serviços de assistência para IST e laboratórios de apoio local) e constatou alta resistência da bactéria à tetracilina, à penicilina e ao ciprofloxacino. ${ }^{18}$

Em 2018, iniciou-se nova fase do Projeto SenGono, com a instalação de mais sítios de coleta e a inclusão de dois novos antimicrobianos (espectinomicina e gentamicina) nas análises. No âmbito do Projeto SenGono, estão sendo pesquisados Neisseria gonorrhoeae, Chlamydia trachomatis, Mycoplasma genitaliume e Trichomonasvaginalis, nas amostras de corrimento uretral; e vírus do herpes simples tipos 1 e 2, Treponema pallidum e Haemophilusducreyi, nas amostras de úlceras genitais. ${ }^{19}$

Os resultados do Projeto SenGono levaram à publicação, em 17 de junho de 2020, da Portaria GM/MS $\mathrm{n}^{0} 1.553 / 2020$, pela qual o Ministério da Saúde instituiu a vigilância sentinela da síndrome do corrimento uretral masculino para monitorar dados em unidades de saúde habilitadas. ${ }^{20}$

Considerando-se o desabastecimento mundial e nacional de penicilina e a dificuldade para sua aquisição pelos estados e municípios no período entre 2014 e 2016, a publicação da 'Agenda de Ações Estratégicas 


\begin{tabular}{|c|c|}
\hline \multirow{4}{*}{2020} & $\begin{array}{l}\text { Atualização do Protocolo Clínico e Diretrizes Terapêuticas (PCDT) para Atenção Integral às Pessoas com Infecções Sexualmente Transmissíveis (IST) } \\
\text { pelo grupo de especialistas em IST. }\end{array}$ \\
\hline & $\begin{array}{l}\text { Realização de seminários na web sobre o PCDT de IST, em parceria com a Organização Pan-Americana da Saúde (OPAS) e a Sociedade Brasileira de } \\
\text { Doenças Sexualmente Transmissiveis. }\end{array}$ \\
\hline & Instituição da Vigilância Sentinela da Síndrome do Corrimento Uretral Masculino pela Portaria GM/MS nº 1.553, de 17 de junho de 2020. \\
\hline & $\begin{array}{l}\text { Lançamento dos resultados do sequenciamento completo do genoma de } 548 \text { cepas coletadas na primeira edição do projeto de vigilância da } \\
\text { resistência ao gonococo (SenGono) (2015/2016). }\end{array}$ \\
\hline \multirow{4}{*}{2019} & $\begin{array}{l}\text { Instituição da Coordenação-Geral de Vigilância das IST na estrutura regimental do Departamento de Doenças de Condições Crônicas e IST, da } \\
\text { Secretaria de Vigilância em Saúde (SVS), pelo Decreto no 9.795, de } 17 \text { de maio de } 2019 .\end{array}$ \\
\hline & Reunião com grupo de especialistas para discutir o PCDT de IST que gerou a atual revisão do documento. \\
\hline & Pactuação com as parcerias para renovação da 'Agenda de Açōes Estratégicas para Redução da Sífilis no Brasil'. \\
\hline & Início da implantação da rede nacional de testes moleculares para clamídia e gonococo junto aos Estados e Distrito Federal. \\
\hline \multirow{7}{*}{2018} & $\begin{array}{l}\text { Atualização do guia 'Como prevenir a transmissão vertical de HIV e sífilis no seu município' (parceria com o Fundo das Nações Unidas para a Infância } \\
\text { [Unicef]). }\end{array}$ \\
\hline & Parceria com Conselho Federal de Medicina (CFM) para realização de teste rápido em gestantes, com a publicação da Recomendação CFM no 1/2018. \\
\hline & Ampliação da vacina contra o papilomavírus humano (HPV) para meninos de 11 a 14 anos de idade, no Sistema Único de Saúde (SUS). \\
\hline & $\begin{array}{l}\text { Incorporação da detecção de clamídia e gonococo por biologia molecular no Sistema de Gerenciamento da Tabela de Procedimentos, Medicamentos } \\
\text { e Órteses, Próteses e Materiais Especiais. }\end{array}$ \\
\hline & Início da nova edição do Projeto SenGono. \\
\hline & Início do estudo de fase ll sobre a eficácia clínica da cefixima para tratamento da sífilis ativa em mulheres não grávidas no Brasil. \\
\hline & Publicação da Portaria SCTIC/MS ño 42, de 5 de outubro de 2018, sobre a aprovação da segunda edição do PCDT de IST. \\
\hline \multirow{10}{*}{2017} & Implantação do projeto de resposta rápida à sífilis - 'Projeto Sífilis Não'. \\
\hline & Publicação da 2a edição do 'PCDT para Prevenção da Transmissão Vertical de HIV, sífilis e hepatites virais'. \\
\hline & Aquisição e distribuição de penicilina cristalina de modo centralizado pelo Ministério da Saúde. \\
\hline & Inclusão de medicamentos para IST na Relação Nacional de Medicamentos Essenciais. \\
\hline & Atualização do tratamento da infecção gonocócica anogenital não complicada (uretra, colo do útero e reto). \\
\hline & Lançamento de dados inéditos nacionais de susceptibilidade do gonococo aos antimicrobianos no âmbito do Projeto SenGono. \\
\hline & $\begin{array}{l}\text { Instituição do } 3^{0} \text { sábado do mês de outubro como'Dia Nacional de Combate à Sífilis e à Sífilis Congênita', Projeto de Lei aprovado pela Câmara dos } \\
\text { Deputados, oficializado pela Lei no } 13.430 \text {, de } 31 \text { de março de 2017, sancionada pelo presidente da República. }\end{array}$ \\
\hline & Início da incorporação no SUS da vacina contra HPV para meninos de 12 e 13 anos de idade. \\
\hline & $\begin{array}{l}\text { Ampliação no SUS da vacina contra HPV para meninos e homens vivendo com HIV de } 9 \text { a } 26 \text { anos de idade, pessoas submetidas a transplantes de } \\
\text { órgãos sólidos, de medula óssea e pacientes oncológicos. }\end{array}$ \\
\hline & Apresentação de resultados preliminares do Estudo Epidemiológico sobre a Prevalência Nacional de Infecção pelo HPV (Estudo POP-Brasil). \\
\hline \multirow{9}{*}{2016} & Lançamento da 'Agenda de Ações Estratégicas para Redução da Sífilis Congênita no Brasil'. \\
\hline & Publicação do 'Manual Técnico para o Diagnóstico da Sífilis', aprovado pela Portaria GM/MS nº 2.012, de 19 de outubro de 2016. \\
\hline & $\begin{array}{l}\text { Substituição/atualização do termo doença sexualmente transmissível (DST) por IST, na definição do então Departamento de Vigilância, Prevenção e } \\
\text { Controle das IST, do HIV/Aids e das Hepatites Virais. }\end{array}$ \\
\hline & Atualização do Álbum Seriado das IST para profissionais de saúde. \\
\hline & $\begin{array}{l}\text { Parceria com Conselho Federal de Enfermagem (Cofen) para realização de testagem rápida de HIV, sífilis e hepatites virais, com a publicação da } \\
\text { Decisão Cofen n⿳0 244/2016. }\end{array}$ \\
\hline & Lançamento do painel de indicadores e dados básicos de sífilis (sífilis em gestantes e sífilis congênita). \\
\hline & Aquisição e distribuição de penicilina benzatina de forma centralizada, pelo Ministério da Saúde. \\
\hline & $\begin{array}{l}\text { Inclusão da síndrome de corrimento uretral masculino na lista nacional de doenças e agravos a serem monitorados por meio da estratégia de } \\
\text { vigilância em unidades sentinelas. }\end{array}$ \\
\hline & Início do Estudo POP-Brasil. \\
\hline
\end{tabular}

\section{Figura 1 - Marco histórico da resposta às infecções sexualmente transmissíveis no Brasil, 1986-2020}


Continuação

\begin{tabular}{|c|c|}
\hline \multirow{7}{*}{2015} & Publicação do primeiro PCDT para Atenção Integral às Pessoas com IST. \\
\hline & Publicação do primeiro PCDT para Prevenção da Transmissão Vertical de HIV, Sífilis e Hepatites Virais. \\
\hline & Publicação do 'Caderno de Boas Práticas sobre Uso de Penicilina na Atenção Primária à Saúde'. \\
\hline & $\begin{array}{l}\text { Parceria com o Cofen para ampliar a administração de penicilina na atenção básica em saúde, pela equipe de enfermagem, com a publicação da } \\
\text { Decisão Cofen nº 0094/2015. }\end{array}$ \\
\hline & Nova edição e ampliação do Projeto SenGono. \\
\hline & Ampliação no SUS da vacina contra HPV para meninas e mulheres de 9 a 26 anos de idade vivendo com HIV. \\
\hline & Descentralização dos testes rápidos de sífilis para unidades da atenção primária à saúde e maternidades. \\
\hline \multirow{4}{*}{2014} & Publicação do 'Protocolo de Investigação de Transmissão Vertical'. \\
\hline & Atualização do curso de diagnóstico de sífilis no programa de educação permanente em diagnóstico das IST (Telelab). \\
\hline & Incorporação no SUS da vacina contra HPV para meninas entre 9 e 13 anos. \\
\hline & Realização do Fórum de Consulta Pública Nacional das DST. \\
\hline 2012 & Implementação de testes rápidos de sífilis e HIV na rotina do pré-natal. \\
\hline 2011 & Instituição da Rede Cegonha. \\
\hline 2010 & Sífilis adquirida passou a ser de notificação compulsória. \\
\hline \multirow{2}{*}{2009} & $\begin{array}{l}\text { Programa Nacional de DST/Aids torna-se Departamento de Doenças Sexualmente Transmissíveis, Aids e Hepatites Virais, subordinado à SVS/ } \\
\text { Ministério da Saúde. }\end{array}$ \\
\hline & Incorporação de teste diagnósticos de clamídia e gonorreia na tabela de procedimentos do SUS. \\
\hline \multirow{2}{*}{2008} & Publicação do guia 'Como prevenir a transmissão vertical de HIV e sífilis no seu município', em parceria com a Unicef. \\
\hline & Publicação da pesquisa sobre 'Prevalências e frequências relativas de DST em populações selecionadas de seis capitais brasileiras, 2005'. \\
\hline \multirow{3}{*}{2007} & Publicação do 'Plano Operacional para a Redução da Transmissão Vertical do HIV e da Sífilis'. \\
\hline & Publicação do 'Protocolo para a Prevenção de Transmissão Vertical de HIV e Sífilis'. \\
\hline & Primeiras iniciativas da implantação do Projeto SenGono. \\
\hline \multirow{3}{*}{2006} & Publicação do 'Manual de Controle das DST' (4a edição) e do 'Manual de Bolso de Controle da Sífilis Congênita'. \\
\hline & Publicação do 'Álbum Seriado das DST'. \\
\hline & Publicação do 'Caderno de Atenção Básica 18: HIV/Aids, Hepatites e outras DST'. \\
\hline \multirow{2}{*}{2005} & Sífilis em gestantes passou a ser agravo de notificação compulsória. \\
\hline & Criação da Rede Nacional de Vigilância da Resistência do Gonococo. \\
\hline 2004 & Inclusão dos dados do venereal disease research laboratory (VDRL) no Sistema de Informações Hospitalares do SUS (SIH/SUS). \\
\hline 2003 & Atualização da definição de caso de sífilis congênita. \\
\hline 1999 & Publicação da $3^{a}$ edição do 'Manual de Controle das DST'. \\
\hline 1998 & Primeira iniciativa nacional do Ministério da Saúde para determinação da susceptibilidade do gonococo aos antimicrobianos. ${ }^{38}$ \\
\hline \multirow{2}{*}{1997} & Publicação da $2^{\text {a }}$ edição do 'Manual de Controle das DST'. \\
\hline & Implantação do sistema Telelab. \\
\hline 1996 & Convite da OPAS-OMS para instituição de rede laboratorial de vigilância de resistência ao gonococo. \\
\hline \multirow{2}{*}{1993} & Publicação da 1a edição do 'Manual de Controle das DST'. \\
\hline & Publicação das 'Bases Técnicas para Eliminação da Sífilis Congênita.' \\
\hline \multirow{2}{*}{1986} & Criação do então Programa Nacional de DST e Aids (PN-DST/Aids). \\
\hline & Sífilis congênita passou a ser de notificação compulsória. \\
\hline
\end{tabular}

Figura 1 - Marco histórico da resposta às infecções sexualmente transmissíveis no Brasil, 1986-2020 
para Redução da Sífilis no Brasil' em 2016, ${ }^{21}$ como resposta à epidemia decretada pelo Ministério da Saúde, teve destaque na decisão pela aquisição centralizada desses medicamentos, reservada ao mesmo Ministério da Saúde. As penicilinas benzatina e cristalina para tratamento da sífilis passaram a fazer parte do componente estratégico da assistência farmacêutica no país. Também foi ampliado o uso da doxiciclina para tratamento de sífilis, donovanose e doença inflamatória pélvica. ${ }^{22} \mathrm{~A}$ referida escassez global de penicilina levou à busca de alternativas eficazes para tratamento de sífilis. Como exemplo, em 2018, iniciou-se a fase II de ensaio clínico para avaliar a eficácia da cefixima no tratamento da sífilis ativa em mulheres não grávidas, estabelecer opções alternativas terapêuticas seguras e apoiar esforços para eliminação da sífilis congênita. ${ }^{23}$

A partir da incorporação da vacinação contra 0 HPV no SUS, observou-se a necessidade de monitorar o impacto dessa imunização na população específica. As pesquisas sobre prevalência do HPV no Brasil eram escassas, até então. ${ }^{10} \mathrm{Em} 2016$, iniciou-se estudo nacional de prevalência da infecção pelo HPV (Estudo POP-Brasil), uma parceria do Ministério da Saúde, entre outras instituições, com a Associação Hospitalar Moinhos de Vento de Porto Alegre, RS. A pesquisa buscou determinar a prevalência do HPV em pessoas sexualmente ativas, de 16 a 25 anos de idade, em todas as capitais brasileiras, além de investigar diferenças regionais de prevalência e tipos de vírus; seus resultados finais suprirão a lacuna de informação epidemiológica, e contribuirão para estabelecer uma linha de base e avaliar o impacto da vacinação contra $0 \mathrm{HPV}$. Os resultados preliminares da pesquisa, trazidos a público ainda em 2016, estimaram alta prevalência de HPV (54,6\%), sendo 38,4\% de HPV de alto risco para o desenvolvimento de câncer. ${ }^{24}$

\section{Desafios e lacunas para controle das IST}

0 rápido crescimento da resistência antimicrobiana da Neisseria gonorrboeae a múltiplos antibióticos ameaça os esforços para controle dessa infecção. Um total de $66 \%$ dos 67 países participantes do Gonococcal Antimicrobial Surveillance Programme já apresentaram elevação da resistência clínica e in vitro às cefalosporinas de espectro estendido, entre $2009 \mathrm{e}$
2014, sendo essa a única monoterapia de primeira linha restante para controle da gonorreia..$^{25} \mathrm{~A}$ evolução das pesquisas deve ter prioridade, incluir o desenvolvimento de novos antimicrobianos para tratamento, da vacina contra o gononoco e de novos testes rápidos, com detecção simultânea de gonococo e resistência antimicrobiana, para diagnóstico e vigilância. ${ }^{26}$

No controle da sífilis são elencados alguns objetivos desafiantes, incluindo: eliminar a transmissão vertical; melhorar a vigilância dos casos; desenvolver testes com maior acurácia, para diagnosticar sífilis ativa, neurossífilis e sífilis congênita; ampliar 0 acesso das populações mais vulneráveis; e desenvolver alternativas de medicamentos orais e vacinas contra 0 Treponema pallidum. ${ }^{27}$

A falta de conhecimento e a circulação de notícias falsas sobre imunizações são exemplos de fatores que contribuem para coberturas vacinais baixas na vacinação contra HPV. A integração dos serviços de saúde com escolas e comunidades é importante para atingir melhores indicadores, haja vista adolescentes bem informados constituírem potenciais transmissores dessas informações aos pais. ${ }^{28,29}$ Também é relevante promover a informação, educação e comunicação em saúde voltada a profissionais da área, visando ampliar as coberturas vacinais. ${ }^{28,30}$

\section{Considerações finais}

Persistem desafios e lacunas a serem superados, na formulação e implementação de políticas públicas em IST no Brasil. Nesse contexto, há necessidade permanente de (i) fortalecer o papel da atenção primária à saúde na atenção integral às pessoas com IST e suas parcerias sexuais, (ii) garantir cobertura vacinal adequada contra a infecção pelo HPV, hepatites virais A e B, (iii) promover informação, educação e comunicação em saúde, (iv) ampliar o acesso à testagem e tratamento das IST, com foco nas populações mais vulneráveis, (v) notificar parcerias sexuais e (vi) qualificar a abordagem de aspectos da saúde sexual pelos profissionais de saúde, para além do rastreamento de assintomáticos, prevenção, manejo clínico-laboratorial e vigilância dos casos de infecção sexualmente transmissível. 


\section{Referências}

1. Rowley J, Vander Hoorn S, Korenromp E, Low N, Unemo M, Abu-Raddad LJ, et al. Chlamydia, gonorrhoea, trichomoniasis and syphilis: global prevalence and incidence estimates, 2016. Bull World Heal Organ [Internet]. 2019 Aug [cited 2020 Sep 10];97(8):548-62. Available from: https://dx.doi.org/10.2471\%2FBLT.18.228486

2. World Health Organization - WHO. Global health sector strategy on sexually transmitted infections 2016-2021 [Internet]. Genebra: World Health Organization; 2016 [cited 2020 Sep 10]. 64 p. Available from: http://www.who.int/ reproductivehealth/publications/rtis/ghss-stis/en/

3. Organização Pan-Americana de Saúde - OPAS. Organização Mundial da Saúde - OMS. Plano de ação para a prevenção e o controle do HIV e de infecções sexualmente transmissíveis [Internet]. Washington, D.C.: OPAS, OMS; 2016 [citado 2020 jun 14]. 401 p. Disponível em: http://iris.paho. org/xmlui/bitstream/handle/123456789/34077/ CD552017-por.pdf?sequence $=1 \&$ isAllowed $=\mathrm{y}$

4. Brasil. Ministério da Saúde. Portaria de Consolidação MS/GM n. 4, de 28 de setembro de 2017. Consolidação das normas sobre os sistemas e os subsistemas do Sistema Único de Saúde [Internet]. Diário Oficial da União, Brasília (DF), 2017 out 3 [citado 2020 set 10];Suplemento:288. Disponível em: http://bvsms.saude.gov.br/ bvs/saudelegis/gm/2017/prc0004_03_10_2017.html

5. Ministério da Saúde (BR). Secretaria de Vigilância em Saúde. Departamento de Doenças de Condições Crônicas e Infecções Sexualmente Transmissíveis. Sífilis 2019. Bol Epidemiol [Internet]. 2019 out [citado 2020 set 10];especial. Disponível em: http://www.aids.gov.br/ pt-br/pub/2019/boletim-epidemiologico-sifilis-2019

6. Pinto VM, Szwarcwald CL, Baroni C, Stringari LL, Inocêncio LA, Miranda AE. Chlamydia trachomatis prevalence and risk behaviors in parturient women aged 15 to 24 in Brazil. Sex Transm Dis [Internet]. 2011 0ct [cited 2020 Sep 10];38(10):957-61. Available from: https://doi.org/10.1097/olq.0b013e31822037fc

7. Barbosa MJ, Moherdaui F, Pinto VM, Ribeiro D, Cleuton M, Miranda AE. Prevalence of neisseria gonorrhoeae and chlamydia trachomatis infection in men attending STD clinics in Brazil. Rev Soc Bras Med Trop [Internet]. 2010 Sep-0ct [cited 2020 Sep 10];43(5):500-3. Available from: http:// dx.doi.org/10.1590/S0037-86822010000500005
8. Miranda AE, Silveira MF, Travassos AG, Tenório T, Val ICC, Lannoy L, et al. Prevalence of chlamydia trachomatis and neisseria gonorrhea and associated factors among women living with Human Immunodeficiency Virus in Brazil: a multicenter study. Braz J Infect Dis [Internet]. 2017 Jul-Aug [cited 2020 Sep 10];21(4):402-7. Available from: http://dx.doi.org/10.1016/j.bjid.2017.03.014

9. Miranda AE, Silveira MF, Travassos AG, Tenório T, Val ICC, Lannoy L, et al. High-risk papillomavirus infection among women living with human immunodeficiency virus: brazilian multicentric study. J Med Virol [Internet]. 2017 Dec [cited 2020 Sep 10];89(12):2217-23. Available from: https://doi.org/10.1002/jmv.24906

10. Colpani V, Falcetta FS, Bidinotto AB, Kops NL, Falavigna $\mathrm{M}$, Hammes LS, et al. Prevalence of human papillomavirus (HPV) in Brazil: A systematic review and meta-analysis. PLoS One [Internet]. 2020 Feb [cited 2020 Sep 10];15(2):e00229154. Available from: https://doi.org/10.1371/journal.pone.0229154

11. Motta LR, Sperhacke RD, Adami AG, Kato SK, Vanni AC, Paganella MP, et al. Syphilis prevalence and risk factors among young men presenting to the Brazilian Army in 2016: results from a national survey. Medicine (Baltimore) [Internet]. 2018 Nov [cited 2020 Sep 10];97(47):e13309. Available from: https://doi.org/10.1097/md.0000000000013309

12. Cunha CB, Friedman RK, Boni RB, Gaydos C, Guimarães MR, Siqueira BH, et al. Chlamydia trachomatis, neisseria gonorrhoeae and syphilis among men who have sex with men in Brazil. BMC Public Health [Internet]. 2015 Jul [cited 2020 Sep 10];15:686. Available from: https://doi.org/10.1186/s12889-015-2002-0

13. Ferreira-Júnior ODC, Guimarães MDC, Damacena GN, Almeida WS, Souza-Júnior PRB, Szwarcwald CL, et al. Prevalence estimates of HIV, syphilis, hepatitis $\mathrm{B}$ and $\mathrm{C}$ among female sex workers (FSW) in Brazil, 2016. Medicine (Baltimore) [Internet]. 2018 May [cited 2020 Sep 10];97(1S Suppl 1):S3-8. Available from: https://doi.org/10.1097/md.0000000000009218

14. Correa ME, Croda J, Castro ARCM, Oliveira SMVL, Pompilio MA, Souza R0, et al. High prevalence of treponema pallidum infection in Brazilian prisoners. Am J Trop Med Hyg [Internet]. 2017 0ct [cited 2020 Sep 10];97(4):1078-84. Available from: https://dx.doi.org/10.4269\%2Fajtmh.17-0098 
15. Brasil. Ministério da Saúde. Secretaria de Ciência, Tecnologia, Inovação e Insumos Estratégicos em Saúde. Portaria MS/SCTIE no 53, de 10 de outubro de 2015. Torna pública a decisão de aprovar o Protocolo Clínico e Diretrizes Terapêuticas (PCDT) de infecções sexualmente transmissíveis no âmbito do Sistema Único de Saúde - SUS [Internet]. Diário Oficial da União, Brasília (DF), 2015 out 5 [citado 2020 set 10];Seção I:691. Disponível em: http://bvsms.saude.gov.br/bvs/ saudelegis/sctie/2015/prt0053_01_10_2015.html

16. Brasil. Presidência da República. Casa Civil. Lei no 12.401, de 28 de abril de 2011. Altera a Lei no 8.080 , de 19 de setembro de 1990, para dispor sobre a assistência terapêutica e a incorporação de tecnologia em saúde no âmbito do Sistema Único de Saúde - SUS [Internet]. Diário Oficial da União, Brasília (DF), 2011 abr 29 [citado 2020 set 10]:Seção I. Diponível em: http://www.planalto.gov.br/ ccivil_03/_Ato2011-2014/2011/Lei/L12401.htm

17. Brasil. Ministério da Saúde. Portaria MS/SCTIE $n^{\circ} 42$, de 5 de outubro de. Torna pública a decisão de aprovar o Protocolo Clínico e Diretrizes Terapêuticas para Atenção Integral às Pessoas com Infecções Sexualmente Transmissíveis (IST), no âmbito do Sistema Único de Saúde - SUS [Internet]. Diário Oficial da União, Brasília (DF), 2018 out 8 [citado 2020 set 10];Seção I:88. Disponível em: http://bvsms.saude.gov.br/bvs/ saudelegis/sctie/2018/prt0042_08_10_2018.html

18. Bazzo ML, Golfetto L, Gaspar PC, Pires AF, Ramos MC, Franchini M, et al. First nationwide antimicrobial susceptibility surveillance for Neisseria gonorrhoeae in Brazil, 2015-16. J Antimicrob Chemother [Internet]. 2018 Jul [cited 2020 Sep 10];73(7):1854-61. Available from: https://doi.org/10.1093/jac/dky090

19. Ministério da Saúde (BR). Secretaria de Vigilância em Saúde. Vigilância da resistência do gonococo aos antimicrobianos. Bol Epidemiol [Internet]. 2020 jul [citado 2020 set 10];51(2). Disponível em: https://www.saude.gov.br/images/pdf/2020/July/09/ Boletim-epidemiologico-SVS-27-06.07.2020.pdf

20. Brasil. Ministério da Saúde. Portaria MS/GM no 1.553, de 17 de junho de 2020. Altera a portaria de consolidação no 5/GM/MS, de 28 de setembro de 2017, para instituir a Vigilância Sentinela da Síndrome do Corrimento Uretral Masculino (VSCUM) [Internet]. Diário Oficial da União, Brasília (DF), 2020 jun 18 [citado 2020 set 10];Seção I:61. Disponível em: http://bvsms.saude.gov.br/bvs/ saudelegis/gm/2020/prt1553_18_06_2020.html
21. Ministério da Saúde (BR). Secretaria de Vigilância em Saúde. Departamento de Vigilância, Prevenção e Controle das Doenças Sexualmente Transmissíveis do HIV/ Aids e das Hepatites Virais. Agenda de ações estratégicas para redução da sífilis congênita no Brasil [Internet]. Brasília: Ministério da Saúde; 2017 [citado 2020 set 10]. 34 p. Disponível em: http://www.aids.gov.br/ system/tdf/pub/2017/65000/agenda_sifilis_10_2017. pdf?file $=1 \&$ type $=$ node $\&$ id $=65000 \&$ force $=1$

22. Ministério da Saúde (BR). Secretaria de Ciência, Tecnologia, Inovação e Insumos Estratégicos em Saúde. Departamento de Assistência Farmacêutica e Insumos Estratégicos. Relação nacional de medicamentos essenciais: RENAME 2017 [Internet]. Brasília: Ministério da Saúde; 2017 [citado 2020 set 10]. 210 p. Disponível em: https://bvsms.saude.gov.br/bvs/publicacoes/ relacao_nacional_medicamentos_rename_2017.pdf

23. Taylor MM, Kara E0, Alix M, Araujo L, Silveira MF, Miranda AE, et al. Phase II trial evaluating the clinical efficacy of cefixime for treatment of active syphilis in non-pregnant women in Brazil (CeBra). BMC Infect Dis [Internet]. 2020 [cited 2020 Sep 10];20:405. Available from: 10.1186/s12879-020-04980-1

24. Wendland EM, Caierão J, Domingues C, Maranhão AGK, Souza FMA, Hammes LS, et al. POP-Brazil study protocol: a nationwide cross-sectional evaluation of the prevalence and genotype distribution of human papillomavirus (HPV) in Brazil. BMJ Open [Internet]. 2018 [cited 2020 Sep 10];8(6). Available from: https://doi.org/10.1136/bmjopen-2017-021170

25. Wi T, Lahra MM, Ndowa F, Bala M, Dillon JR, Ramon-pardo P, et al. Antimicrobial resistance in neisseria gonorrhoeae: global surveillance and a call for international collaborative action. PLoS One [Internet]. 2017 Jul [cited 2020 Sep 10];14(7):e1002344. Available from: https://doi.org/10.1371/journal.pmed.1002344

26. Unemo M, Lahra MM, Cole M, Galarza P, Ndowa F, Martin I, et al. World Health Organization Global Gonococcal Antimicrobial Surveillance Program (WHO GASP): review of new data and evidence to inform international collaborative actions and research efforts. Sex Health [Internet]. 2019 Sep [cited 2020 Sep 10];16(5):412-25. Available from: https://doi.org/10.1071/sh19023

27. Peeling RW, Mabey D, Kamb ML, Chen X-S, Radolf JD, Benzaken AS. Syphilis. Nat Rev Dis Prim [Internet]. 2017 0ct [cited 2018 May 8];3:17073. Available from: https://doi.org/10.1038/nrdp.2017.73 
28. Domingues CMAS, Fantinato FFST, Duarte E, Garcia LP. Vacina Brasil e estratégias de formação e desenvolvimento em imunizações. Epidemiol Serv Saúde [Internet]. 2019 out [citado 2020 ago 10];28(2):e20190223. Disponível em: https:// doi.org/10.5123/s1679-49742019000200024

29. Succi RCM. Recusa vacinal - que é preciso saber. J Pediatr (Rio de Janeiro) [Internet]. 2018 nov-dez [citado 2020 set 10];94(6):574-81. https://dx.doi.org/10.1016/j.jped.2018.01.008

30. Oliveira CM, Fregnani JHTG, Villa LL. HPV vaccine: updates and highlights. Acta Cytol [Internet]. 2019 [cited 2020 Sep 10];63(2):159-68. Available from: https://doi.org/10.1159/000497617

Recebido em 27/07/2020

Aprovado em 08/09/2020

Editora associada: Taís Freire Galvão - (1) orcid.org/0000-0003-2072-4834 